\title{
Octamer-binding protein 4 affects the cell biology and phenotypic transition of lung cancer cells involving $\beta$-catenin/E-cadherin complex degradation
}

\author{
ZHONG-SHU CHEN ${ }^{1,2^{*}}$, DONG-JIN LING ${ }^{1,2^{*}}$, YANG-DE ZHANG ${ }^{1}$, JIAN-XIONG FENG ${ }^{2}$, \\ XUE-YU ZHANG ${ }^{2}$ and TIAN-SHENG SHI ${ }^{2}$ \\ ${ }^{1}$ Ministry of Health Hepatobiliary and Enteric Surgery Center, Xiangya School of Medicine, \\ Central South University, Changsha, Hunan 410008; ${ }^{2}$ Department of Thoracic Surgery, \\ Jiangxi Provincial Chest Hospital, Nanchang, Jiangxi 330006, P.R. China
}

Received April 9, 2014; Accepted August 6, 2014

DOI: $10.3892 / \mathrm{mmr} .2014 .2992$

\begin{abstract}
Clinical studies have reported evidence for the involvement of octamer-binding protein 4 (Oct4) in the tumorigenicity and progression of lung cancer; however, the role of Oct4 in lung cancer cell biology in vitro and its mechanism of action remain to be elucidated. Mortality among lung cancer patients is more frequently due to metastasis rather than their primary tumors. Epithelial-mesenchymal transition (EMT) is a prominent biological event for the induction of epithelial cancer metastasis. The aim of the present study was to investigate whether Oct 4 had the capacity to induce lung cancer cell metastasis via the promoting the EMT in vitro. Moreover, the effect of Oct4 on the $\beta$-catenin/E-cadherin complex, associated with EMT, was examined using immunofluorescence and immunoprecipitation assays as well as western blot analysis. The results demonstrated that Oct4 enhanced cell invasion and adhesion accompanied by the downregulation of epithelial marker cytokeratin, and upregulation of the mesenchymal markers vimentin and $\mathrm{N}$-cadherin. Furthermore, Oct4 induced EMT of lung cancer cells by promoting $\beta$-catenin/E-cadherin complex degradation and regulating nuclear localization of $\beta$-catenin. In conclusion, the present study indicated that Oct4 affected the cell biology of lung cancer cells in vitro through promoting lung cancer cell metastasis via EMT; in addition, the results suggested that the association and degradation of
\end{abstract}

Correspondence to: Professor Yang-De Zhang, Ministry of Health Hepatobiliary and Enteric Surgery Center, Xiangya School of Medicine, Central South University, 87 Xiangya Road, Changsha, Hunan 410008, P.R. China

E-mail: ydzhangxy@163.com

*Contributed equally

Key words: lung cancer, $\beta$-catenin/E-cadherin degradation, octamer-binding protein 4 the $\beta$-catenin/E-cadherin complex was regulated by Oct 4 during the process of EMT.

\section{Introduction}

Lung cancer is one of the most prevalent types of malignant tumor worldwide, with a five-year survival rate of $\sim 16 \%$ (1). Mortality among lung cancer patients is more frequently due to metastasis rather than their primary tumors. Therefore, it is necessary to elucidate the molecular mechanisms of lung cancer metastasis in order to develop effective treatment options.

Epithelial-mesenchymal transition (EMT) is a process characterized by downregulation of epithelial markers and upregulation of mesenchymal markers $(2,3)$. Previous studies have proposed that EMT may be a key step in the progression of tumor cell metastasis (4-6).

Octamer-binding protein 4 (Oct4), a transcription factor that belongs to the Pit-Oct-Unc (POU) family, has been reported to be a master regulator of maintenance and differentiation in pluripotent cells. It has been suggested that Oct4 may be a key component of the regulation of self-renewal and differentiation in stem cells (7-9); in addition, Oct4 may also have a crucial role in cancer development (10). Chen et al (11) demonstrated that Oct4 expression was involved in the tumorigenesis and malignancy of lung cancer. The aims of the present study were to investigate the effect of Oct4 on the cell biology of lung cancer cells in vitro, elucidate the underlying mechanisms associated with lung cancer metastasis and examine the effect of Oct4 on the degradation of the $\beta$-catenin/E-cadherin complex degradation, a process strongly associated with EMT.

\section{Materials and methods}

Cell culture. A549 cells were purchased from the American Type Culture Collection (Manassas, VA, USA). Cells were cultured in Dulbecco's modified Eagle's medium (DMEM; Invitrogen Life Technologies, Carlsbad, CA, USA) with 10\% fetal bovine serum (FBS; Invitrogen Life Technologies) at $37^{\circ} \mathrm{C}$ in a $5 \% \mathrm{CO}_{2}$ humidified atmosphere. 
Tissues. Tumor and adjacent normal lung tissue specimens were collected from ten patients with non-small cell lung cancer at Jiangxi Provincial Chest Hospital (Nanchang, China). This study was approved by the Ethics Committee of Jiangxi Provincial Chest Hospital. All patients provided written informed consent in compliance with the code of ethics of the World Medical Association (Declaration of Helsinki; Ferney-Voltaire, France). None of the ten patients had received chemotherapy or radiotherapy prior to surgery. The tumor and adjacent normal tissue specimens were frozen in liquid nitrogen directly following surgery and stored at $-80^{\circ} \mathrm{C}$ until further use.

Constructs and transfection. An open reading frame clone of homo Oct4 was subcloned into enhanced green fluorescent protein plasmind-C1 (pEGFP-C1) vector (Invitrogen Life Technologies). Small hairpin RNA (shRNA) targeting Oct4 was designed and inserted into pGPU6/GFP/Neo vector (Invitrogen Life Technologies). The plasmids pEGFP-C1-Oct4 and shRNA-Oct4 were transfected into A549 cells using Lipofectamine 2000 (Invitrogen Life Technologies) according to the manufacturer's instructions.

MTT assay. Cells were seeded in 96-well plates at a density of $1 \times 10^{5} / \mathrm{ml}$ and allowed to grow at $37^{\circ} \mathrm{C}$ in a $5 \% \mathrm{CO}_{2}$ humidified atmosphere. $10 \mu \mathrm{l}$ MTT reagent (Sigma-Aldrich, St. Louis, MO, USA) was added to each well and incubated at $37^{\circ} \mathrm{C}$ for $4 \mathrm{~h}$. The formazan dye was solubilized in $150 \mu \mathrm{l}$ dimethyl sulfoxide and the absorbance was measured at $570 \mathrm{~nm}$ using a microplate reader (Multiskan Ascent 354; Thermo Labsystems, Waltham, MA, USA).

Flow cytometry. Cells were dual-stained with Alexa Fluor 488-Annexin V and propidium iodide (PI) using an Annexin V-fluorescein isothiocyanate/PI apoptosis kit (Kaiji Biological Inc., Nanjing, China) according to the manufacturer's instructions. The apoptotic rate was measured using flow cytometry (FC 500 MPL system; Beckman Coulter Inc., Miami, FL, USA).

Immunoprecipitation. Cells were extracted with immunoprecipitation lysis buffer (Beyotime, Shanghai, China). The cell lysates were incubated with an anti-E-cadherin antibody or normal immunoglobulin $\mathrm{G}(\mathrm{IgG})$, followed by a recombinant fusion of protein A and protein $\mathrm{G}$ agarose (Sigma). Following centrifugation at $1000 \mathrm{x}$ g for $5 \mathrm{~min}$ and washing with immunoprecipitation lysis buffer (Beyotime, Shanghai, China) five times, proteins were analyzed using SDS-PAGE.

Cell invasion assay. The cell invasion assay was carried out using a Transwell chamber (Corning Inc., Corning, NY, USA) pre-coated with Matrigel ${ }^{\circledR}$ (BD Biosciences, Franklin Lakes, NJ, USA). $5 \times 10^{4}$ A549 cells were added to the upper chamber of the Transwell plates (Corning Inc., Corning, NY, USA) with serum-free medium (DMEM; Invitrogen Life Technologies) and the lower chamber was filled with $1 \mathrm{ml} \mathrm{DMEM}$ containing $10 \%$ FBS. Following $12 \mathrm{~h}$ of incubation at $37^{\circ} \mathrm{C}$, cells in the upper chamber were removed using a cotton swab, the invaded cells were fixed using $95 \%$ ethanol (Xinchenghuagong Inc., Guangzhou, China) for $15 \mathrm{~min}$ and then stained with hematoxylin (Maixin, Fuzhou, China) for $10 \mathrm{~min}$. The invaded cells were then counted under a light microscope (TS100, Nikon, Tokyo, Japan).

Cell adhesion assay. A 96-well-plate was precoated with fibronectin (Sigma) for $2 \mathrm{~h}$. The wells were washed with phosphate-buffered saline (PBS; Maixin) and then blocked with $1 \%$ bovine serum albumin (BSA; Amresco Inc., Solon, $\mathrm{OH}$, USA) for $2 \mathrm{~h}$. Cells were seeded in the wells at a final concentration of $3 \times 10^{5}$ cells $/ \mathrm{ml}$ in serum-free medium. Following $2 \mathrm{~h}$ of incubation, the wells were washed with PBS and the cells were fixed in paraformaldehyde (Xinchenghuagong Inc., Guangzhou, China). The number of adherent cells was quantified using the colorimetric MTT assay.

Quantitative polymerase chain reaction ( $q P C R)$. Total RNA from cells or tissue specimens was isolated using Trizol reagent (Invitrogen Life Technologies). Complementary DNA (cDNA) was synthesized by a First Strand cDNA Synthesis kit (Fermentas, Vilnius, Lithuania) using $1 \mu \mathrm{g}$ RNA template. qPCR was performed in a 7300 Sequence Detection System (Applied Biosystems, Foster City, CA, USA) using a $\mathrm{SYBR}^{\circledR}$ Green PCR kit (Applied Biosystems). The relative expression levels of each messenger RNA (mRNA) were calculated using comparative computerized tomography (CT) methods (SDS Software version 1.4.1; Applied Biosystems) with GAPDH as an internal control.

Western blot analysis. Whole cell extracts were obtained using a total protein extraction kit (Promab, Changsha, China), $\beta$-catenin nuclear and cytoplasmic fractions were obtained using a nuclear and cytoplasmic extraction kit (Beyotime, Shanghai, China). $50 \mu$ g protein was separated using 10\% SDS-PAGE and transferred onto a nitrocellulose membrane (Millipore, Billerica, MA, USA). Membranes were blocked with $5 \% \mathrm{BSA}$ at room temperature for $1 \mathrm{~h}$. Membranes were then washed with PBS and incubated with the corresponding primary antibody [rabbit polyclonal to Oct4 (1:400), rabbit polyclonal to $\beta$-catenin (1:500), mouse monoclonal to histone (1:1000) and mouse monoclonal to GAPDH (1:800) (Santa Cruz Biotechnology Inc., Santa Cruz, CA, USA); rabbit monoclonal to vimentin (1:1000; Abcam, Cambridge, MA, USA); rabbit polyclonal to $\mathrm{N}$-cadherin (1:400), rabbit polyclonal to E-cadherin (1:400) and rabbit polyclonal to pan-cytokeratin (1:400) (Signalway Antibody Inc., College Park, MD, USA)] overnight at $4^{\circ} \mathrm{C}$, then incubated with horseradish peroxidase-conjugated secondary antibodies [Goat Anti Mouse IgG/HRP(1:40000) and Goat Anti Rabbit IgG/HRP(1:40000);Santa Cruz Biotechnology, Inc.)] for $1 \mathrm{~h}$ at room temperature. Bands were detected using an enhanced chemiluminesence detection kit (Pierce, Rockford, IL, USA). GAPDH expression was used as an internal control.

Immunofluorescence. Cell slides were washed with PBS three times, then fixed in $4 \%$ paraformaldehyde solution at $4{ }^{\circ} \mathrm{C}$ for $1 \mathrm{~h}$. Following fixation, slides were washed again with PBS at room temperature and permeabilized by exposure to $0.2 \%$ Triton X-100 (Sigma-Aldrich) at $4^{\circ} \mathrm{C}$ for $1 \mathrm{~h}$. Slides were blocked with goat serum (Maixin) at $4^{\circ} \mathrm{C}$ for $1 \mathrm{~h}$, then 
A

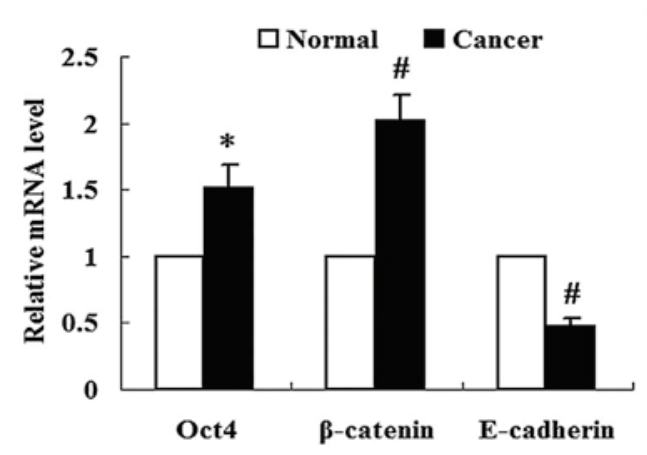

B

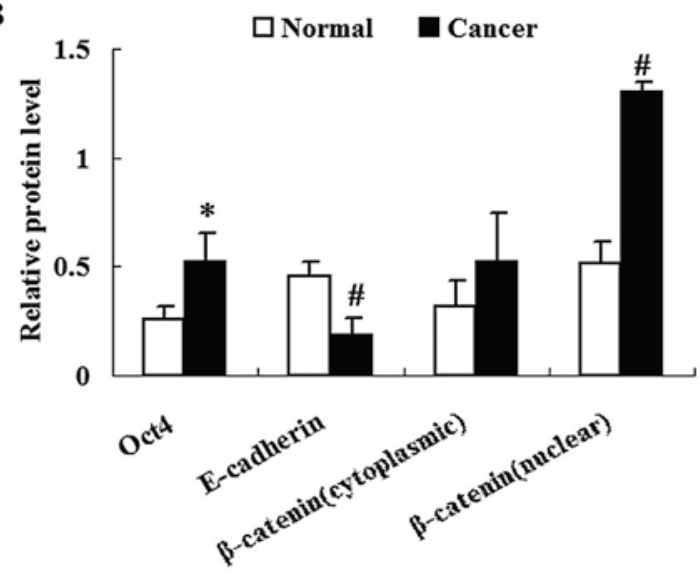

Figure 1. mRNA and protein expression levels of Oct4, E-cadherin and nuclear $\beta$-catenin are altered in lung cancer compared with those in adjacent normal tissues. (A) Relative mRNA levels of Oct $4, \beta$-catenin and E-cadherin, normalized to GAPDH. (B) Relative protein levels of Oct4, $\beta$-catenin and E-cadherin, normalized to GAPDH. ${ }^{~} \mathrm{P}<0.05$ and ${ }^{\prime} \mathrm{P}<0.01$ vs. normal group. Oct 4 , octamer-binding protein 4 . Data are presented as the mean \pm standard deviation.

A

B
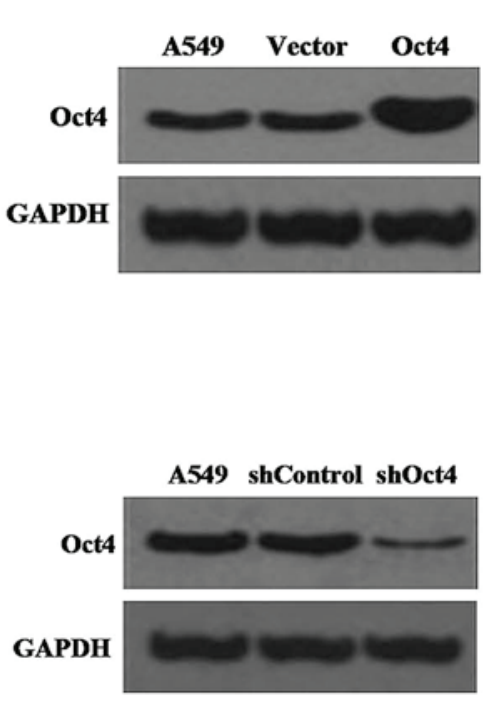
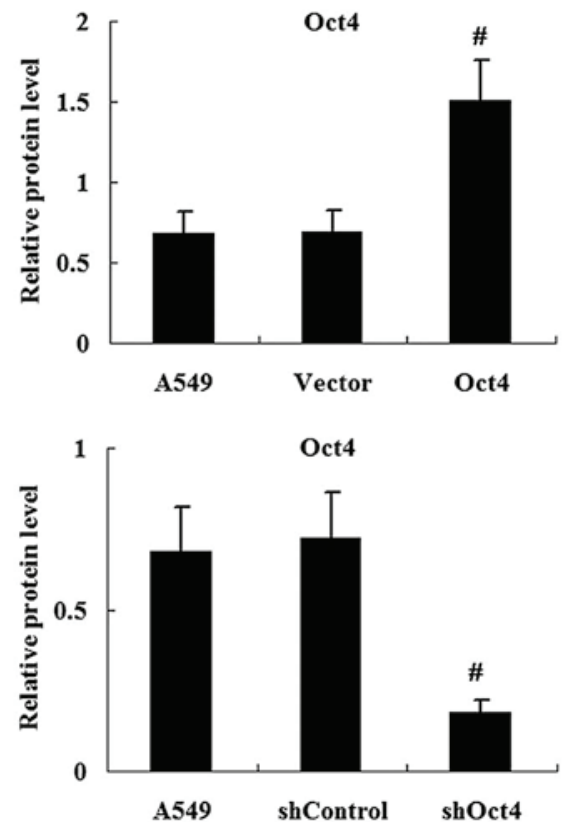

Figure 2. Transfection with pEGFP-C1-Oct4 and shOct4 have opposite effects on the relative protein levels of Oct 4 in A549 cells. Western blot analysis of the relative protein levels of Oct 4 following transfection with (A) pEGFP-C1 vector and pEGFP-C1-Oct4; and (B) shControl and shOct4. ${ }^{*} \mathrm{P}<0.01$ vs. control group. Oct4, octamer-binding protein 4; shRNA, small hairpin RNA; pEGFP-C1, enhanced green fluorescent protein plasmind-C1; shControl, control shRNA; shOct 4 , Oct 4 shRNA. Data are presented as the mean \pm standard deviation.

incubated with primary antibodies [mouse monoclonal to E-cadherin (1:400) and rabbit monoclonal to $\beta$-catenin (1:400); Abcam] for $1 \mathrm{~h}$ at $37^{\circ} \mathrm{C}$. Slides were washed again with PBS and secondary antibodies [Alexa Fluor 555-labeled Goat anti-Rabbit IgG (1:200) or Alexa Fluor488 labeled Goat anti mouse IgG (1:200); Abcam] were added, slides were then incubated at $37^{\circ} \mathrm{C}$ for $1 \mathrm{~h}$. DAPI (Invitrogen Life Technologies) was used to label the nucleus and slides were visualized under a fluorescence microscope (80i, Nikon).

Statistical analysis. SPSS 19.0 statistical software (IBM corp., Armonk, NY, USA) was used for statistical analysis. All data represent the mean \pm standard deviation of at least three inde- pendent experiments. Statistical differences were analyzed using the Student's t-test. $\mathrm{P}<0.05$ was considered to indicate a statistically significant difference between values.

\section{Results}

Expression of Oct4 is increased in lung cancer tissues. Expression of Oct4 in lung cancer tissues was analyzed using qPCR and western blot analysis. Results from qPCR and western blot analysis revealed increased expression of Oct4 mRNA and relative protein levels, respectively, in lung cancer tissues compared with those in the adjacent normal tissues $(\mathrm{P}<0.05)$ (Fig. 1A and $\mathrm{B})$. 
A

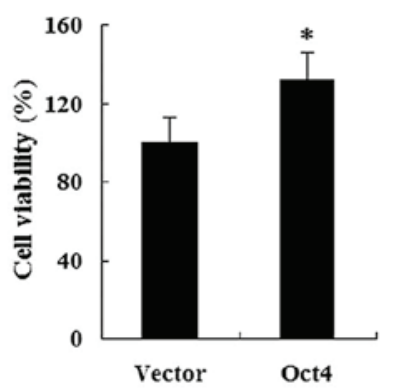

C

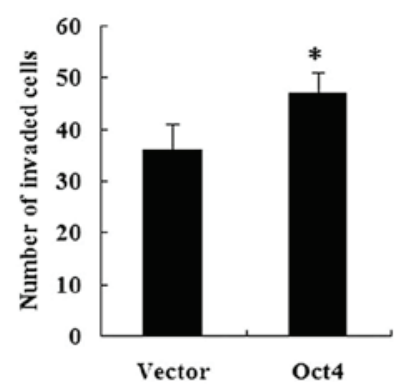

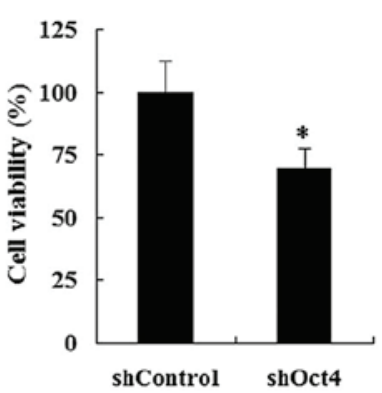

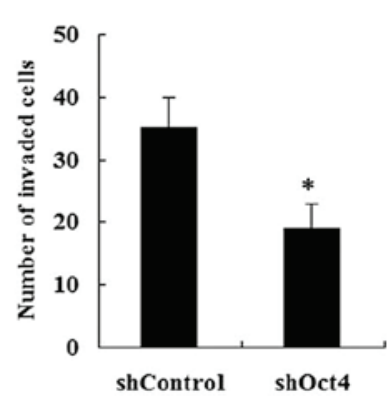

B

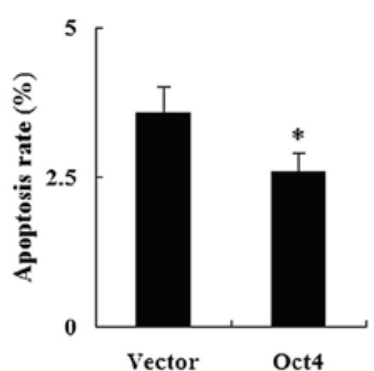

D

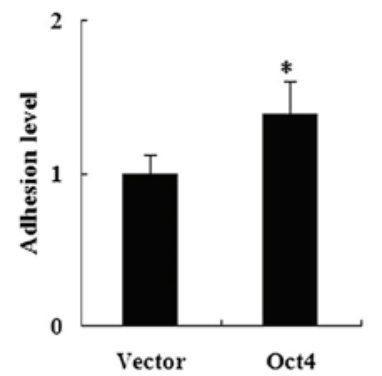

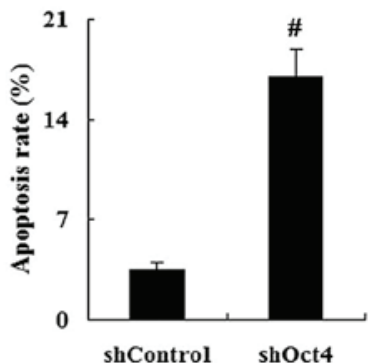

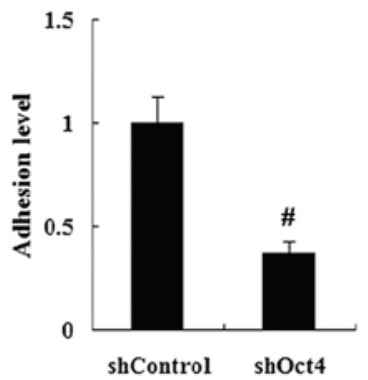

Figure 3. Transfection with pEGFP-C1-Oct4 and shOct4 alters the cell biology of A549 cells in vitro. (A) Cell viability; (B) cell apoptosis rate; (C) invaded cell numbers; and (D) adhesion ability of A549 cells following transfection with pEGFP-C1-Oct4 and shOCT4 compared with pEGFP-C1 vector and shControl, respectively. ${ }^{*} \mathrm{P}<0.05$ and ${ }^{*} \mathrm{P}<0.01$ vs. control group. Oct4, octamer-binding protein 4; shRNA, small hairpin RNA; pEGFP-C1, enhanced green fluorescent protein plasmind-C1; shControl, control shRNA; shOct4, Oct 4 shRNA. Data are presented as the mean \pm standard deviation.

A

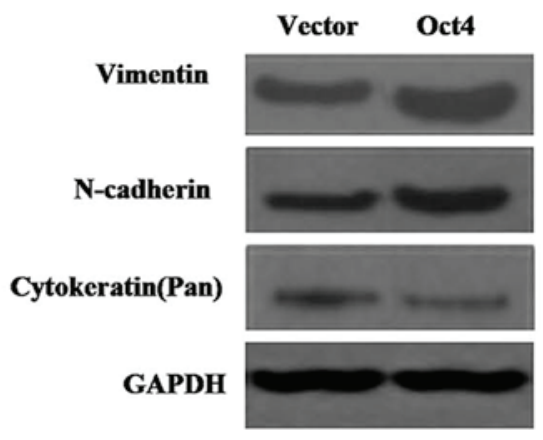

B

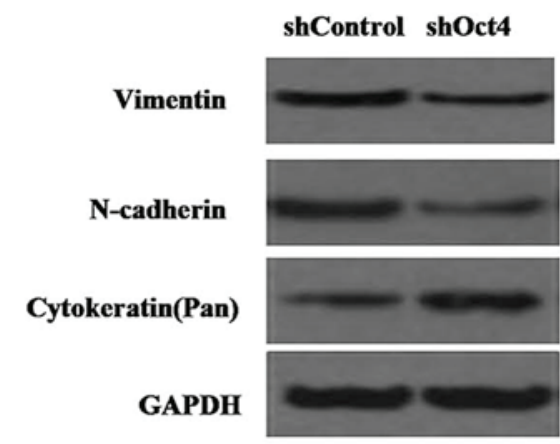

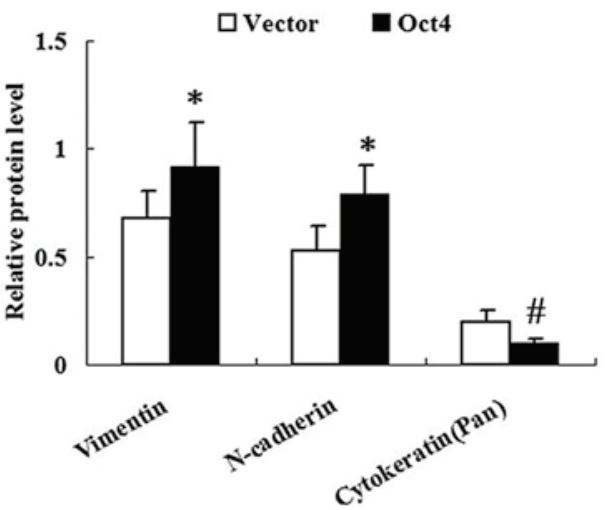

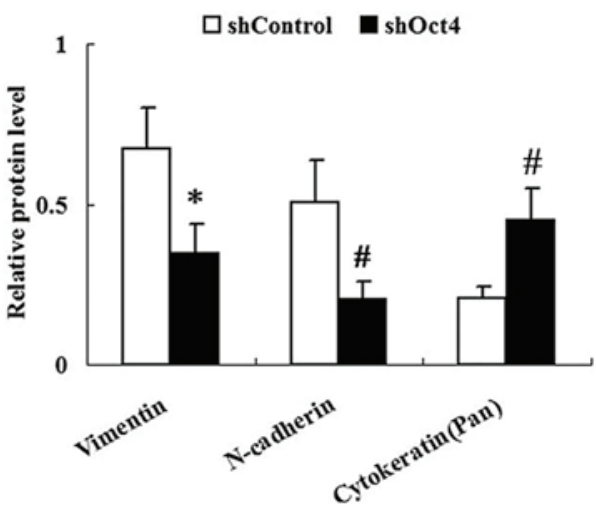

Figure 4. Transfection with pEGFP-C1-Oct4 and shOct4 alters the expression levels of mesenchymal and epithelial cell markers in A549 cells. Western blot analysis of the expression levels of mesenchymal cell markers vimentin and $\mathrm{N}$-cadherin, as well as the epithelial cell marker cytokeratin in A549 cells following transfection with (A) pEGFP-C1-Oct4 and (B) shOct4. ${ }^{*} \mathrm{P}<0.05$ and ${ }^{~} \mathrm{P}<0.01$ vs. control group. Oct4, octamer-binding protein 4; shRNA, small hairpin RNA; pEGFP-C1, enhanced green fluorescent protein plasmind-C1; shControl, control shRNA; shOct4, Oct 4 shRNA. Data are presented as the mean \pm standard deviation.

Expression of $\beta$-catenin and E-cadherin is altered in lung cancer tissues. As shown in Fig. 1A, $\beta$-catenin mRNA expression was significantly increased in lung cancer tissues compared with that in adjacent normal tissues $(\mathrm{P}<0.05)$. Nuclear and cytoplasmic fractions of $\beta$-catenin were collected from the respective tissues. Western blot analysis revealed 


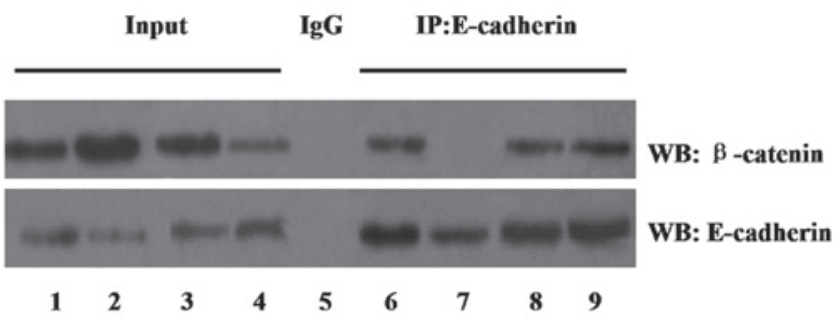

Figure 5. Effect of Oct4 on the association and degradation of the $\beta$-catenin/E-cadherin complex determined by co-immunoprecipitation assay. Input lanes represent the total cell lysates. IgG was used as a control. Lanes: 1 and 6, vector group; 2 and 7, Oct4 group; 3 and 8, shRNA-control group; 4 and 9, shRNA-Oct4 group; 5, combination of the four groups. IP, immunoprecipitaion; WB, western blot; Oct4, octamer-binding protein 4; shRNA, small hairpin RNA; IgG, immunoglobulin G.

A

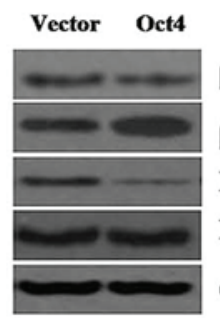

$\beta$-catenin (cytoplasmic)
$\beta$-catenin (nuclear)
E-cadherin
Histone
GAPDH

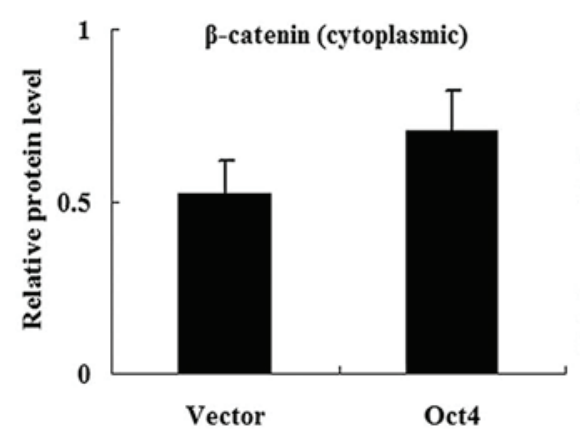

B
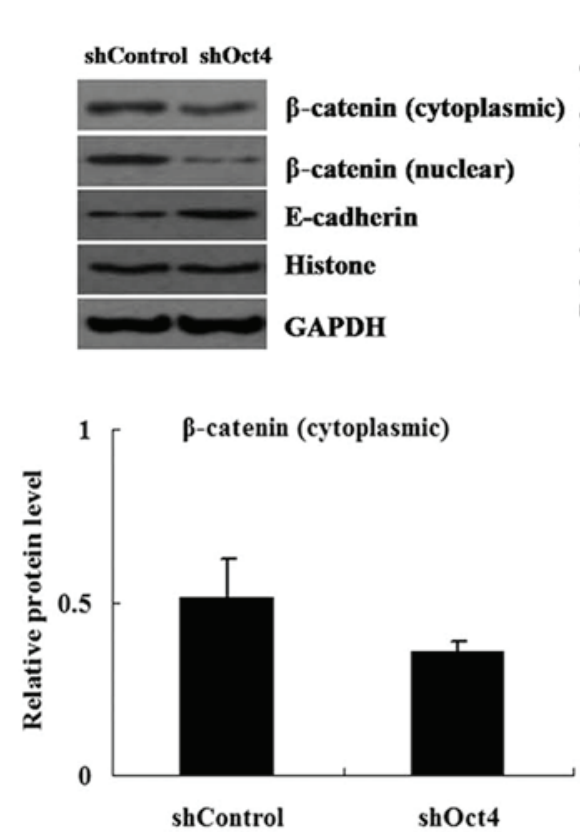
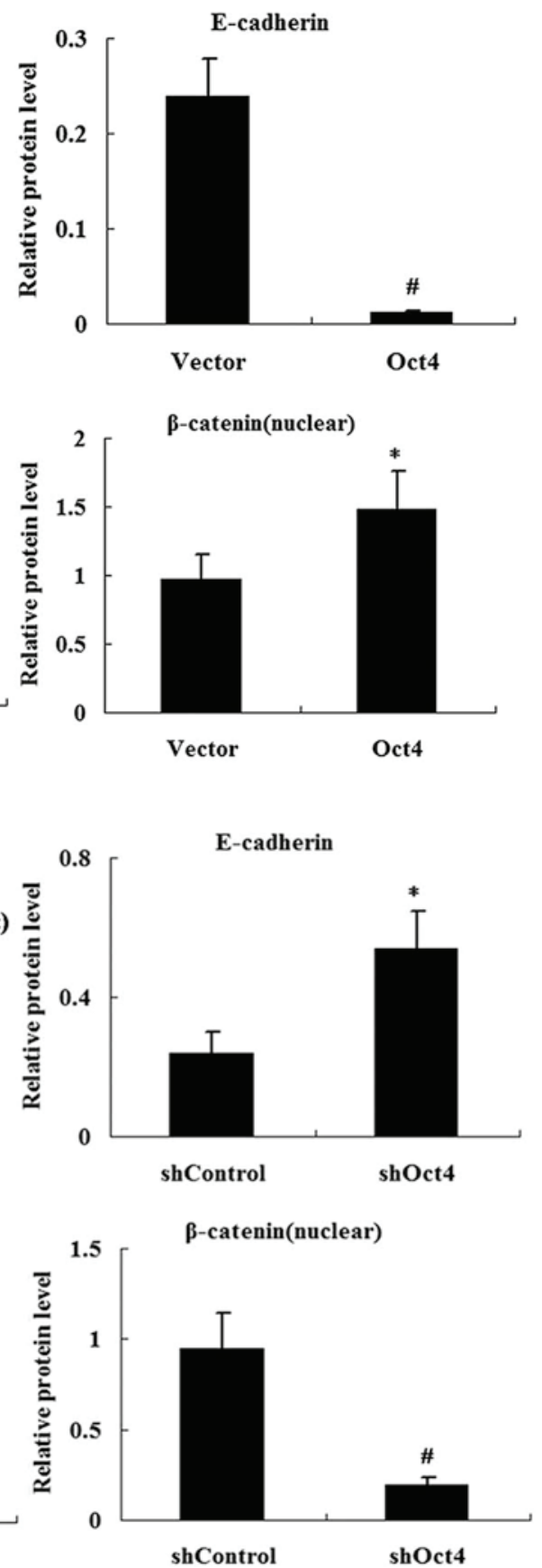

Figure 6. Opposite effects of transfection with pEGFP-C1-Oct4 and shOct4 on the $\beta$-catenin/E-cadherin complex. Relative protein levels of $\beta$-catenin (nuclear) normalized to histone, $\beta$-catenin (cytoplasmic) and E-cadherin normalized to GAPDH in A549 cells when transfected with (A) pEGFP-C1-Oct4 to induce Oct 4 overexpression; or (B) shOct4 to repress Oct 4 expression. ${ }^{*} \mathrm{P}<0.05$ and ${ }^{\#} \mathrm{P}<0.01$ vs. control group. Oct 4 , octamer-binding protein 4 ; shRNA, small hairpin RNA; shControl, control shRNA; shOct4, Oct 4 shRNA. Data are presented as the mean \pm standard deviation. 


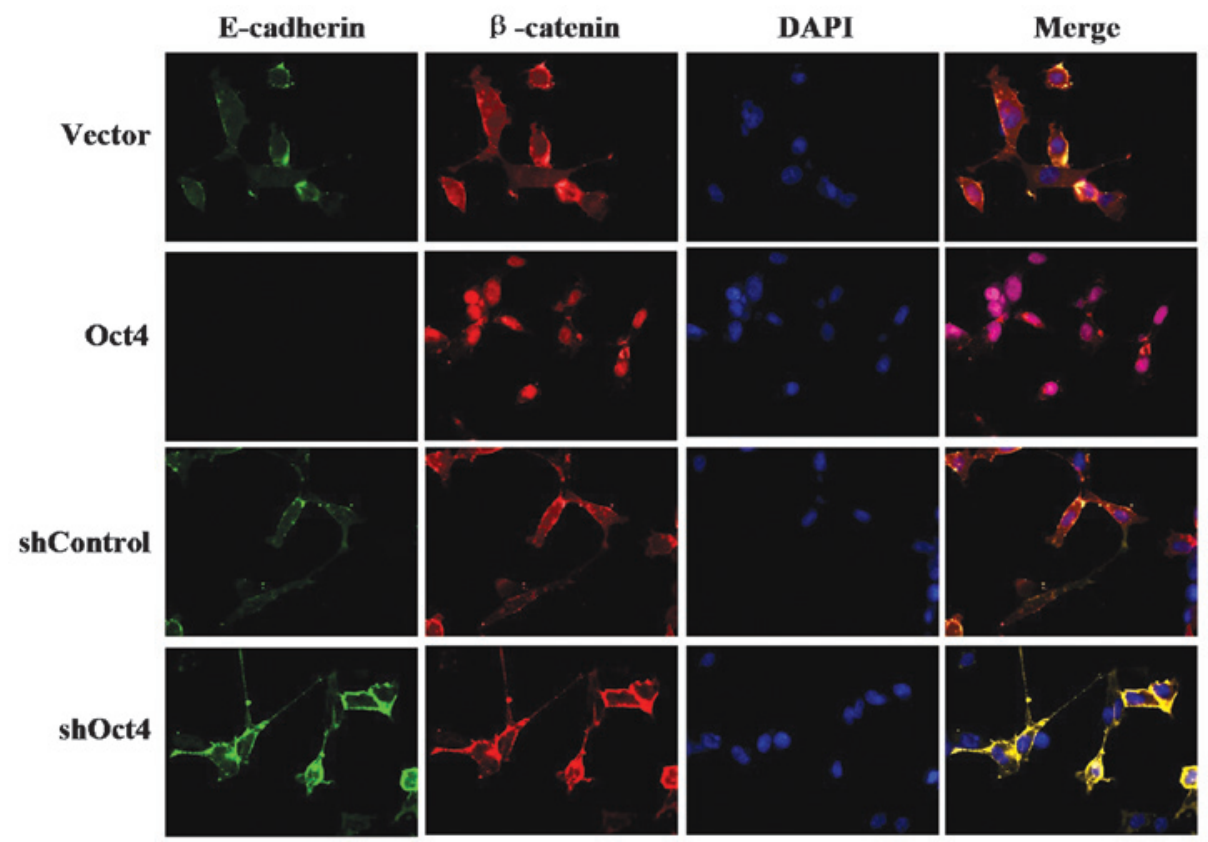

Figure 7. Immunofluorescence staining of $\beta$-catenin (red) and E-cadherin (green) in Oct4 overexpressing and shOct 4 transfected A549 cells. Nuclear DNA was stained with DAPI (blue) (magnification, x400). Oct4, octamer-binding protein 4; shRNA, small hairpin RNA; shControl, control shRNA; shOct4, Oct 4 shRNA.

that the protein expression levels of cytoplasmic $\beta$-catenin in lung cancer tissues were not significantly different from those of adjacent normal tissues ( $\mathrm{P}>0.05$ ) (Fig. 1B). By contrast, protein expression levels of nuclear $\beta$-catenin were significantly increased in lung cancer tissues compared with those of adjacent normal tissues $(\mathrm{P}<0.01)$ (Fig. 1B).

The expression of E-cadherin was significantly reduced in lung cancer tissues compared with that of adjacent normal tissues at the mRNA and protein level $(\mathrm{P}<0.01)$ (Fig. 1).

Changes in expression levels of Oct4 in pEGFP-C1-Oct4 and shRNA-Oct4-transfected cells. In order to efficiently enhance or repress Oct 4 expression, A549 cells were transfected with pEGFP-C1-Oct4 or shRNA-Oct4. Western blot analysis was used to measure the protein expression levels of Oct4 in pEGFP-C1-Oct4- and shRNA-Oct4-transfected cells. The results demonstrated that the expression levels of Oct4 protein were increased in pEGFP-C1-Oct4 transfected cells compared with those in the pEGFP-C1 vector transfected cells $(\mathrm{P}<0.01)$ (Fig. 2A). As hypothesized, the shRNA-Oct 4 transfected cells showed decreased expression of Oct4 protein in comparison to that of the shRNA-Control transfected cells $(\mathrm{P}<0.01)$ (Fig. 2B).

Opposite effects of overexpression and repression of Oct4 on cell viability, apoptosis, invasion and adhesion in vitro. The effect of Oct4 on cell biology was examined in A549 cells using an MTT assay to analyze cell proliferation. As shown in Fig. 3A, Oct4 overexpression significantly increased cell viability, whereas repression of Oct 4 significantly reduced cell viability in vitro. Furthermore, the apoptotic rate in the Oct4 overexpression group was significantly decreased compared with that of the vector control group, whereas shRNA-Oct4 had the reverse effect (Fig. 3B). Cell invasion assays revealed that Oct4 overexpression resulted in an increased number of invasive cells in comparison to that of the vector control. However, in the shRNA-Oct4 group, the number of invasive cells was significantly decreased compared with that of the shRNA-control group (Fig. 3C). Cell adhesion assays revealed that the adhesive activity in the Oct4 overexpression group was significantly increased compared with that of the vector control group. Following transfection with shRNA-Oct4, the adhesion activity of the A549 cells was reduced compared with that of the shRNA-control (Fig. 3D).

Changes in expression of Oct4 alter the expression levels of phenotypic transition markers in A549 cells. The effect of Oct4 on EMT-like phenotypic changes in A549 cells was examined. Western blot analysis was performed in order to determine the expression levels of vimentin, $\mathrm{N}$-cadherin and cytokeratin in A549 cells. As shown in Fig. 4, the expression levels of vimentin and $\mathrm{N}$-cadherin were significantly increased in the pEGFP-C1-Oct4-transfected group but decreased in the shRNA-Oct 4 group. By contrast, expression of cytokeratin was decreased in the pEGFP-C1-Oct4-transfected group, but in the shRNA-Oct4 group cytokeratin expression was increased compared with that in the vector control group.

Changes in expression of Oct4 alters the expression of the $\beta$-catenin/E-cadherin complex. In order to examine the effect of Oct4 on the association and degradation of the $\beta$-catenin/E-cadherin complex, cell lysates were immunoprecipitated with an anti-E-cadherin antibody and western blot analysis was performed with an anti- $\beta$-catenin antibody. Equal protein was confirmed in each group using a western blot with anti-E-cadherin antibody.

The co-immunoprecipitation study, shown in Fig. 5, demonstrated that the degradation of $\beta$-catenin with E-cadherin was increased in the Oct4 overexpression group 
compared with that of the vector control group. However, the association of $\beta$-catenin with E-cadherin was enhanced in the shRNA-Oct4 group.

As shown in Fig. 6, relative protein levels of E-cadherin were decreased in the Oct4 overexpression group; however, they were increased in the shRNA-Oct 4 group compared with those of the control groups. Nuclear and cytoplasmic fractions of $\beta$-catenin were obtained from A549 cells. Western blot analysis demonstrated that $\beta$-catenin nuclear protein levels were significantly increased in the Oct 4 overexpression group but decreased in the shRNA-Oct4 group. The expression of cytoplasmic $\beta$-catenin did not alter significantly in the Oct 4 overexpression group or shRNA-Oct4 group compared with that of the control group.

In addition, an immunofluorescence assay was used to examine the localization of $\beta$-catenin and E-cadherin. The results of the assay demonstrated induced nuclear localization of $\beta$-catenin in the Oct 4 overexpression group; however, in the shRNA-Oct4 group, $\beta$-catenin was primarily localized in cytoplasm. Furthermore, the immunofluorescence assay revealed a decrease in membrane localization of E-cadherin in the Oct4 overexpression group compared with that in the control group, but increased expression of membrane E-cadherin in the shRNA-Oct4 group (Fig. 7).

\section{Discussion}

Oct4 is a transcription factor that has been shown to be highly expressed in embryonic stem (ES) cells and essential for the induction of somatic cell pluripotency (7). In addition, Oct 4 has been implicated in various human cancers and was reported to be associated with tumor progression or bad prognosis (12-17). The present study demonstrated that the mRNA and protein expression of Oct4 was higher in human lung cancer tissues than that in adjacent normal tissues. In addition, Oct4 was able to affect the cell biology of lung cancer cells by inducing cell proliferation, inhibiting apoptosis as well as promoting cell invasion and adhesion. Numerous clinical studies have examined the expression of Oct4 in lung cancer patients (18-20); however, the molecular mechanisms of its oncogenic role remain to be elucidated.

The majority of newly diagnosed lung cancer patients have locally invasive cancer, and almost all of these patients go on to develop metastatic disease, which accounts for most cancer-associated mortalities worldwide (21). In order to investigate the role of Oct4 in lung cancer cell metastasis, the present study examined whether Oct 4 could regulate cell invasion and adhesion in vitro using A549 cells. The results indicated that increased expression of Oct4 led to enhanced cell invasion and adhesion abilities. Conversely, repression of Oct 4 demonstrated the opposite effect, therefore indicating that Oct4 promoted lung cancer cell metastasis.

Epithelial-mesenchymal transition (EMT) has been shown to be an important process for the metastatic progression of epithelial cancer $(3,22)$. Therefore, the present study aimed to investigate whether Oct4 had a role in the regulation of EMT in lung cancers. During EMT, epithelial cell-derived cancer cells lose their epithelial properties and acquire mesenchymal properties (23). Vimentin, a member of the intermediate filament family, is an important canonical marker of EMT (24), as it was reported to induce changes in cell shape, motility and adhesion during the EMT (25). $\mathrm{N}$-cadherin was reported to be involved in the metastasis of cancer cells indicated by the association between abnormal $\mathrm{N}$-cadherin expression, the acquisition of the EMT phenotype and the enhanced invasive properties of lung cancer cell lines (26). The remodeling of the cytoskeleton has been suggested to be a hallmark of EMT. Loss of cytokeratins leads to alterations in cell-to-cell adhesions and changes in polarity and cell motility (27). The results of the present study showed that Oct4 upregulated the expression of the mesenchymal markers vimentin and $\mathrm{N}$-cadherin, as well as downregulated the expression of the epithelial marker cytokeratin in A549 cells. These results indicated that Oct4 induced lung cancer cell metastasis via the mechanism of EMT.

$\beta$-catenin/E-cadherin association has an essential role in the regulation and provision of cellular adhesion (28). $\beta$-catenin interacts with E-cadherin by binding directly to its cytoplasmic tail, therefore creating a bridge between E-cadherin and the actin cytoskeleton, which stabilizes the adherence junction (29). Nuclear $\beta$-catenin has been suggested to have a pivotal role in tumor progression $(30,31)$. The results of the present study demonstrated the downregulation of E-cadherin as well as the upregulation of nuclear $\beta$-catenin protein in lung cancer tissues, therefore indicating that nuclear $\beta$-catenin acts as an oncogenic protein in lung cancer. EMT is controlled by several transcription factors, which may be able to suppress E-cadherin promoter activity and repress E-cadherin expression $(32,33)$. It has been suggested that nuclear $\beta$-catenin can induce Slug or Twist 1 gene expression (34), which may lead to the further repression of E-cadherin and thereby contribute to EMT. $\beta$-catenin/E-cadherin degradation is associated with tumor invasion and metastasis (35). In the present study, immunoprecipitation assays revealed that Oct 4 promoted $\beta$-catenin/E-cadherin degradation in lung cancer cells during EMT. Oct4 was shown to induce EMT of lung cancer cells while repressing E-cadherin expression. In addition, the localization of $\beta$-catenin was examined using western blot and immunofluorescence assays, which demonstrated the upregulation of nuclear $\beta$-catenin protein by Oct 4 .

In conclusion, the results of the present study indicated that Oct4 affected the cell biology of lung cancer cells in vitro, promoted lung cancer cell metastasis through EMT and regulated the $\beta$-catenin/E-cadherin complex during the process of EMT.

\section{References}

1. Jemal A, Siegel R, Ward E, et al: Cancer statistics, 2008. CA Cancer J Clin 58: 71-96, 2008.

2. Foroni C, Broggini M, Generali D and Damia G: Epithelial-mesenchymal transition and breast cancer: Role, molecular mechanisms and clinical impact. Cancer Treat Rev 38: 689-697, 2012.

3. Drasin DJ, Robin TP and Ford HL: Breast cancer epithelial-to-mesenchymal transition: Examining the functional consequences of plasticity. Breast Cancer Res 13: 226, 2011.

4. Jechlinger M, Grünert S and Beug H: Mechanisms in epithelial plasticity and metastasis: insights from 3D cultures and expression profiling. J Mammary Gland Biol Neoplasia 7: 415-432, 2002 
5. Thiery JP: Epithelial-mesenchymal transitions in tumour progression. Nat Rev Cancer 2: 442-454, 2002.

6. Hanahan D and Weinberg RA: Hallmarks of cancer: the next generation. Cell 144: 646-674, 2011.

7. Nichols J, Zevnik B, Anastassiadis K, et al: Formation of pluripotent stem cells in the mammalian embryo depends on the POU transcription factor Oct4. Cell 95: 379-391, 1998.

8. Hay DC, Sutherland L, Clark J and Burdon T: Oct-4 knockdown induces similar patterns of endoderm and trophoblast differentiation markers in human and mouse embryonic stem cells Stem Cells 22: 225-235, 2004.

9. Boiani M and Schöler HR: Regulatory networks in embryo-derived pluripotent stem cells. Nat Rev Mol Cell Biol 6: 872-884, 2005.

10. Monk M and Holding C: Human embryonic genes re-expressed in cancer cells. Oncogene 20: 8085-8091, 2001.

11. Chen YC, Hsu HS, Chen YW, et al: Oct-4 expression maintained cancer stem-like properties in lung cancer-derived CD133-positive cells. PLoS One 3: e2637, 2008.

12. Kim RJ and Nam JS: OCT4 expression enhances features of cancer stem cells in a mouse model of breast cancer. Lab Anim Res 27: 147-152, 2011.

13. Zhang $\mathrm{Y}$, Zhang X, Wang X, et al: Inhibition of LDH-A by lentivirus-mediated small interfering RNA suppresses intestinal-type gastric cancer tumorigenicity through the downregulation of Oct4. Cancer Lett 321: 45-54, 2012.

14. Guo Y, Liu S, Wang P, et al: Expression profile of embryonic stem cell-associated genes Oct4, Sox 2 and Nanog in human gliomas. Histopathology 59: 763-775, 2011.

15. Iida H, Suzuki M, Goitsuka R and Ueno H: Hypoxia induces CD133 expression in human lung cancer cells by up-regulation of OCT3/4 and SOX2. Int J Oncol 40: 71-79, 2012.

16. He W, Li K, Wang F, Qin YR and Fan QX: Expression of OCT4 in human esophageal squamous cell carcinoma is significantly associated with poorer prognosis. World J Gastroenterol 18: 712-719, 2012

17. Schoenhals M, Kassambara A, De Vos J, et al: Embryonic stem cell markers expression in cancers. Biochem Biophys Res Commun 383: 157-162, 2009.

18. Zhang X, Han B, Huang J, Zheng B, Geng Q, Aziz F and Dong Q: Prognostic significance of OCT4 expression in adenocarcinoma of the lung. Jpn J Clin Oncol 40: 961-966, 2010.

19. Moreira AL, Gonen M, Rekhtman N and Downey RJ: Progenitor stem cell marker expression by pulmonary carcinomas. Mod Pathol 23: 889-895, 2010

20. Li X, Wang J, Xu Z, et al: Expression of sox 2 and oct 4 and their clinical significance in human non-small-cell lung cancer. Int J Mol Sci 13: 7663-7675, 2012.

21. Lee W, Jiang Z, Liu J, et al: The mutation spectrum revealed by paired genome sequences from a lung cancer patient. Nature 465: 473477,2010
22. Brabletz T, Hlubek F, Spaderna S, et al: Invasion and metastasis in colorectal cancer: Epithelial-mesenchymal transition, mesen-chymal-epithelial transition, stemcells and beta-catenin. Cells Tissues Organs 179: 56-65, 2005.

23. Kalluri R and Weinberg RA: The basics of epithelial-mesenchymal transition. J Clin Invest 119: 1420-1428, 2009.

24. Satelli A and Li S: Vimentin in cancer and its potential as a molecular target for cancer therapy. Cell Mol Life Sci 68: 3033-3046, 2011.

25. Mendez MG, Kojima S and Goldman RD: Vimentin induces changes incell shape, motility, and adhesion during the epithelial to mesenchymal transition. FASEB J 24: 1838-1851, 2010.

26. Zhang X, Liu G, Kang Y, et al: N-cadherin expression is associated with acquisition of EMT phenotype and with enhanced invasion in erlotinib resistant lung cancer cell lines. PLoS One 8: e57692, 2013

27. König K, Meder L, Kröger C, et al: Loss of the keratin cyto skeleton is not sufficient to induce epithelial mesenchymal transition in a novel KRAS driven sporadic lung cancer mouse model. PLoS One 8: e57996, 2013.

28. Gumbiner B, Stevenson B and Grimaldi A: The role of the cell adhesion molecule uvomorulin in the formation and maintenance of the epithelial junctional complex. J Cell Biol 107: 1575-1587, 1988.

29. Provost E and Rimm DL: Controversies at the cytoplasmic face of the cadherin based adhesion complex. Curr Opin Cell Biol 11: 567-572, 1999

30. Morin PJ, Sparks AB, Korinek V, et al: Activation of beta-catenin-Tcf signaling in colon cancer by mutations in beta catenin or APC. Science 275: 1787-1790, 1997.

31. Korinek V, Barker N, Morin PJ, et al: Constitutive transcrip $\neg$ tional activation by a beta-catenin-Tcf complex in APC-/ colon carcinoma. Science 275: 1784-1787, 1997.

32. Yang J, Mani SA, Donaher JL, et al: Twist, a master regulator of morphogenesis, plays an essential role in tumor metastasis. Cell 117: 927-939, 2004

33. Bolós V, Peinado H, Pérez-Moreno MA, et al: The transcription factor Slug represses E-cadherin expression and induces epithelial to mesenchymal transitions: a comparison with Snail and E47 repressors. J Cell Sci 116: 499-511, 2003.

34. Conacci-Sorrell M, Simcha I, Ben-Yedidia T, et al: Autoregulation of E-cadherin expression by cadherin-cadherin interactions: The roles of beta-catenin signaling, Slug, and MAPK. J Cell Biol 163: 847-857, 2003.

35. Sommers CL, Gelmann EP, Kemler R, et al: Alterations in beta-catenin phosphorylation and plakoglobin expression in human breast cancer cells. Cancer Res 54: 3544-3552, 1994. 